\title{
Aves de Pernambuco: o estado atual do conhecimento ornitológico
}

\author{
Gilmar Beserra de Farias ${ }^{1,2 *}$ \\ Glauco Alves Pereira ${ }^{2}$ \\ ${ }^{1}$ Universidade Federal de Pernambuco, Centro Acadêmico de Vitória, Núcleo de Biologia \\ Rua Alto do Reservatório, s/n, CEP 55.608-680, Vitória de Santo Antão - PE, Brasil \\ ${ }^{2}$ Observadores de Aves de Pernambuco - OAP, Paulista - PE, Brasil \\ *Autor para correspondência \\ gilmarfarias@br.inter.net
}

Submetido em 30/09/2008

Aceito para publicação em 09/03/2009

\section{Resumo}

Uma análise do atual estado do conhecimento ornitológico em Pernambuco pode orientar pesquisadores em suas futuras investigações. O objetivo deste trabalho foi fazer uma revisão bibliográfica sobre o conhecimento ornitológico em Pernambuco. Entre os anos de 1880 e 2008 foram elaboradas diversas listas que contribuíram para a sistematização do conhecimento das aves nesse Estado. Das 535 espécies registradas para Pernambuco, 49 ocorrem em ambientes marinhos ou limícolas, 450 na Mata Atlântica e 270 em regiões de caatinga. Sugerese que estudos sobre aves em áreas de caatinga sejam mais importantes e prioritários nesse momento, além de ações efetivas para a conservação de espécies endêmicas e ameaçadas de extinção.

Unitermos: aves, Caatinga, Mata Atlântica, Pernambuco, Unidades de Conservação

\section{Abstract}

Birds of Pernambuco: current state of ornithological knowledge. An analysis of the present state of ornithological knowledge is required for the guidance of researchers in their future investigations. This work provides a survey of literature describing the development of ornithological research in the state. Between 1880 and 2008, many lists were organized, which contributed towards the systematization of the knowledge about Pernambuco's birds. Out if the 535 species recorded, 49 are found in marine environments or wetlands, 450 occur in the Atlantic Rainforest, and 270 reside in the semi-arid Caatinga. We suggest that studies on the birds of the Caatinga are most important and should be prioritized at present, in addition to effective actions for the conservation of endemic species and of species under risk of extinction.

Key words: Atlantic Forest, birds, Caatinga, Pernambuco, protected areas 


\section{Introdução}

Trabalhos sobre as riquezas naturais dos primeiros séculos da colonização brasileira, nos quais os autores organizaram uma relação de nomes vernáculos locais para os animais e plantas, iniciaram a construção do conhecimento sobre a avifauna no país. As primeiras informações sobre aves no Estado de Pernambuco foram produzidas nesse processo. Durante a ocupação holandesa no Nordeste brasileiro (1630-1645), a convite do príncipe Maurício, Conde de Nassau, o astrônomo George Marcgrave e o médico Guilherme Piso foram os primeiros cientistas a entrar em contato direto com a natureza dessa região (Teixeira, 1992; Pacheco, 2000). Pode-se dizer que foi no Século XVII, principalmente em Pernambuco, Paraíba e no Rio Grande do Norte, que aconteceram os primeiros estudos sistemáticos sobre a fauna regional, incluindo as aves, por meio de obras históricas como Theatrum Rerum Naturalium Brasiliae de George Marcgrave (1942). Neste livro, muitas espécies de aves foram descritas, mas, em sua maioria, não existem informações precisas sobre a procedência dos exemplares.

Buscar dados históricos para melhor compreender a amplitude e os esforços empregados em qualquer área do conhecimento pode evidenciar aos pesquisadores desafios para o futuro (Pacheco, 2000). Assim, uma primeira análise do estado atual do conhecimento ornitológico em Pernambuco pode orientar os pesquisadores na construção de suas futuras investigações, promovendo a continuidade de esforços para revelar novas informações em cenários já estudados, corrigir ou confirmar antigos registros e pesquisar em novas localidades. Dessa forma, o objetivo deste trabalho foi fazer uma revisão bibliográfica dos trabalhos considerados mais relevantes para o desenvolvimento do conhecimento ornitológico em Pernambuco.

\section{As listas de aves em Pernambuco}

Após o período Mauriciano, a primeira contribuição à ornitologia pernambucana foi produzida no início do Século XIX. Em 1816, o naturalista inglês William Swainson visitou o Estado de Pernambuco e desenvolveu inicialmente suas atividades ornitológicas nos arredores de Recife, seguindo até a Bahia coletando material científico. O trabalho de Swainson foi apresentado de forma fragmentada e sem a indicação detalhada das localidades de coleta, o que dificultou o dimensionamento de sua contribuição à ornitologia da região Nordeste (Pacheco, 2000) e, particularmente, para Pernambuco.

Pode-se afirmar que os estudos ornitológicos realizados de forma mais objetiva e sistemática, em Pernambuco, tiveram início por volta de 1880, estabelecendo até os dias de hoje um período de quase 130 anos de produção científica na ornitologia local. A primeira iniciativa para colecionar registros nesse Estado foi feita, em 1880, por um ornitólogo londrino chamado William Forbes (Pinto, 1940), que mesmo não tendo a intenção de fazer uma lista de aves para o estado, organizou um corpo de informações que representou o primeiro levantamento sistematizado das aves de Pernambuco. Realizou excursões pela Zona da Mata e Sertão, coletando espécimes e, posteriormente, encaminhando exemplares para o Museu Britânico, apresentando uma lista com 108 espécies (Forbes, 1881).

Outros trabalhos realizados por naturalistas estrangeiros também contribuíram para os primeiros registros de algumas espécies de aves nesse Estado, principalmente para a região da Caatinga. No final do Século XIX, o entomologista francês Edmond Gounelle coletou beija-flores em localidades próximas de Arcoverde e Águas Belas, no sertão de Pernambuco (Sick, 1997; Pacheco, 2000). Em 1903, o ornitólogo austríaco Otmar Reiser participou de uma expedição ao Nordeste do Brasil, com início em Pernambuco, coletando exemplares em Recife, Paudalho e Petrolina (Reiser, 1925).

Em 1907, o alemão naturalizado brasileiro Hermann von Ihering e o seu filho Rodolpho von Ihering publicaram o Catálogo da Fauna Brazileira (Ihering e Ihering, 1907), no qual o volume primeiro aborda o tema As Aves do Brazil, citando 118 espécies de aves para Pernambuco. Entre 1926 e 1928, o alemão Emil Kaempfer coletou aves na região Nordeste e no interior de Pernambuco, sendo estes exemplares depositados posteriormente no American Museum of Natural History, em Nova Iorque. Muitos registros provenientes dessa 
coleção foram analisados e publicados posteriormente pelos ornitólogos Elsie M. B. Naumburg, John T. Zimmer e Kenneth E. Stager (Pacheco, 2000).

Em 1933, outro trabalho baseado principalmente em referências bibliográficas foi escrito por Theodoro Kadletz (Kadletz, 1933), catedrático alemão e professor do Ginásio Pernambucano, que publicou Lista das aves de Pernambuco (Farias et al., 2000a). Essa lista apresenta pouca utilidade, pois o autor declarou textualmente que confeccionou a lista de aves a partir de obras gerais e acrescentou algumas que ele próprio teria observado, com espécies de improvável ocorrência para o Nordeste (Pacheco, 2000). Possivelmente, algumas dessas espécies foram inseridas erroneamente a partir das peles coletadas pelo naturalista francês Louis Jacques Brunet, em excursões realizadas na Região Norte do Brasil, por volta de 1862 (Rosado e Silva, 1973), e depositadas no Museu do Ginásio Pernambucano, em Recife.

A partir da década de 1940, as informações sobre a avifauna de Pernambuco ficaram mais confiáveis e robustas, pois alguns ornitólogos começaram a publicar listas a partir de espécimes coletados e depositados em museus, além de observações de campo. Entre os anos de 1938 e 1939, Olivério Pinto, ornitólogo do Museu Paulista, colecionou exemplares na Zona da Mata de Pernambuco, realizando saídas de campo nas Matas da Usina São José (Igarassu) e colecionando aves marinhas no litoral de Itamaracá, ao norte do Estado, apresentando uma lista com 212 espécies, publicadas em Aves de Pernambuco (Pinto, 1940).

Entre os anos de 1944 e 1945, Herbert Berla, naturalista do Museu Nacional do Rio de Janeiro, realizou coletas e observações de aves na Zona da Mata pernambucana, incluindo também as matas da Usina São José, e elaborou uma lista com 53 espécies para aquele local (Berla, 1946). Indivíduos de Ramphastos vitellinus foram registrados por Berla como "comuns nos capoeirões da Usina São José”, assim como Lipaugus vociferans, outra importante espécie frugívora. Espécies como Tinamus solitarius, Crypturellus strigulosus e Turdus albicollis foram coletadas e tiveram os seus primeiros registros para Pernambuco nesse trabalho, constituindo-se atualmente em registros raros e/ou únicos destas espécies para o estado (Farias et al., 2007), além de uma subespécie nova para a Ciência, Tinamus solitarius pernambucensis, que recentemente foi incluída em sinonímia com a espécie nominal (Amaral e Silveira, 2004).

De 1943 a 1947, Donald W. Lamm, diplomata norteamericano e membro do American Ornithologists'Union (Speich, 1999), realizou 450 horas de trabalhos de campo relativos à observação e coleta de aves, especialmente na zona da mata de Pernambuco e da Paraíba (Pacheco, 2000). Seu trabalho, publicado no periódico Auk, em 1948, apresentou algumas espécies registradas para as densas florestas do município de Goiana. "Várias viagens foram feitas a uma área de floresta perto da cidade de Goiana, aproximadamente quarenta milhas ao norte de Recife. Foram achadas espécies como cotingas e araçaris, principalmente nesta área" (Lamm, 1948, p.262). Foram registrados por Lamm Harpagus bidentatus, Xipholena atropurpurea, Caryothraustes canadensis, Myiobius atricaudus e Tyrannus savana, espécies novamente observadas em Pernambuco apenas recentemente. Neste trabalho, o autor assinalou outra espécie de importância biogeográfica para a região, Porzana flaviventer, porém o mesmo não citou a localidade nem o estado onde foi realizado o registro.

Em 1960, o jornalista e historiador pernambucano Mário Melo escreveu o artigo Aves de Pernambuco, nomes vulgares e científicos, publicado na Revista do Instituto Arqueológico Histórico e Geográfico Pernambucano (Melo, 1960). O autor não apresentou nenhuma metodologia ou critério de inclusão das espécies, sendo essa lista, provavelmente, construída a partir de informações bibliográficas e do conhecimento local acerca da avifauna. Mário Melo era notório por valorizar o conhecimento popular e, possivelmente, essa lista foi baseada em coletas de informações locais e organizada pelos nomes científicos a partir de listas divulgadas na época, como aquelas publicadas por Pinto (1938; 1940; 1944), por exemplo.

A partir da década de 1990, por meio da associação Observadores de Aves de Pernambuco (OAP), quatro listas de aves foram sistematicamente organizadas, cada uma valorizando um aspecto em particular que as tornaram diferentes, mas sempre com a proposta de atualizar os registros para esse Estado. Essas listas foram 
evoluindo e ficando mais precisas. A Lista preliminar das aves do estado de Pernambuco (Farias et al., 1995) foi construída a partir de todos os registros de campo da OAP, referências bibliográficas e espécimes depositados em museus, principalmente da UFPE e do Parque Zoológico de Dois Irmãos, em Recife. Essa primeira lista tornou-se referência em Ornitologia Brasileira (Sick, 1997), inserida em um conjunto de estados brasileiros que já possuíam essas informações coligidas. Aves de Pernambuco e seus nomes populares (Farias et al., 2000a) acrescentou novos registros, além de reunir os nomes vernáculos locais. Em Registros Ornitológicos de Pernambuco (Farias et al., 2002), além dos acréscimos à lista, as fontes bibliográficas foram citadas, assim como as cidades onde os registros foram feitos. Recentemente, em Lista das aves de Pernambuco (Farias et al., 2008), houve uma organização dos registros baseados em evidencia documental (lista primária com 442 espécies) e de registros sem evidência documental conhecida ou disponível (lista secundária com 93 espécies), totalizando 535 espécies registradas.

Nos últimos anos, um dos fatores que mais contribuíram para o maior conhecimento da ornitofauna pernambucana tem sido a atuação de grupos de birdwatching liderados por guias e pesquisadores experientes. A contribuição desses ornitólogos refletiu-se no maior conhecimento de espécies antes desconhecidas para o Estado e de um maior conhecimento biogeográfico de várias espécies de aves, como o da andorinha migrante do hemisfério norte Petrochelidon pyrrhonota, documentada no oeste de Pernambuco, sendo considerada como um dos primeiros registros para a região Nordeste (Pereira et al., 2008) e o primeiro para a Caatinga.

Assim, no Nordeste do Brasil, Pernambuco é o Estado que tem atualizado periodicamente a sua lista de aves. Isso também se revela a partir de vários artigos publicados frequentemente desde o final da década de 1980 até os dias atuais, descrevendo novas espécies, documentando registros, ampliando a distribuição geográfica e fazendo novas ocorrências para o litoral, Mata Atlântica e a Caatinga (Teixeira et al., 1986; 1987; 1988; 1989; 1993; Farias et al., 1994; Telino-Júnior et al., 2000; Silva et al., 2002; Roda e Carlos, 2003; Roda et al., 2003; Lyra-Neves et al., 2004a; Pereira et al., 2006a; 2006b; 2006c; Roda e Pereira, 2006; Dantas et al., 2007; Pereira et al., 2008).

\section{Aves do litoral, da Mata Atlântica e da caatinga}

No Brasil, existem 148 espécies de aves costeiras e marinhas (Vooren e Brusque, 1999), incluindo muitas espécies migratórias do hemisfério norte e sul. A costa do litoral nordestino faz parte da rota atlântica na migração de diversas espécies do hemisfério norte (Antas, 1983). Em Pernambuco, aves marinhas e limícolasmigratórias são estudadas desde a década de 1980, em pesquisas realizadas pela Universidade Federal Rural de Pernambuco (UFRPE) na Ilha de Itamaracá (AzevedoJúnior, 1992; 1998), principalmente com censo, captura, marcação, recuperação de anilhas, capacidade de vôo, aquisição de massa corpórea, plumagens e mudas (Azevedo-Júnior et al., 2001a; 2001b; 2001c; AzevedoJúnior e Larrazábal, 2002; Telino-Júnior et al., 2003; Lyra-Neves et al., 2004b; Fedrizzi et al., 2004), além de novas ocorrências (Azevedo-Júnior et al., 2003; Carlos et al., 2005). Atualmente, nesse Estado, foram registradas 49 espécies marinhas ou limícolas. Das limícolas, a maioria é migratória do hemisfério norte (Azevedo-Júnior e Larrazábal, 2002). Ficam fora dessa lista Bartramia longicauda e Calidris melanotos, observadas recentemente em Petrolina, no extremo oeste (Pereira et al., 2008).

Em relação à Mata Atlântica, esse é seguramente o bioma com maior número de espécies de aves em Pernambuco. São aproximadamente 450 espécies, $65 \%$ do total registrado para a Mata Atlântica Brasileira (682 espécies, segundo Stotz et al., 1996). Depois de Forbes (1881), Pinto (1940), Berla (1946) e Lamm (1948), o inventário com maior esforço amostral em área de Mata Atlântica foi realizado por Coelho (1979; 1987a), na Estação Ecológica do Tapacurá, em São Lourenço da Mata. A partir daí, muitas pesquisas foram realizadas em outras áreas de Mata Atlântica, incluindo inventários, distribuição de espécies ameaçadas de extinção, ectoparasitismo, levantamentos quantitativos, estrutura trófica, comportamento interespecífico, marcação e recaptura de aves (Roda e Farias, 1999; Lyra-Neves et al., 2000; Silveira et al., 2003; Lyra-Neves et al., 2003; 
Lyra-Neves et al., 2004c; Barnett et al., 2005; TelinoJúnior et al., 2005; Lyra-Neves et al., 2005; Lyra-Neves et al., 2007; Pereira et al., 2007; Dantas et al., 2007; Magalhães et al., 2007; Farias, 2009a). Destacam-se ainda os Relatórios Técnicos do Centro de Pesquisas Ambientais do Nordeste (CEPAN), principalmente aqueles sobre as aves em Unidades de Conservação (UCs) e matas pertencentes a usinas de cana-de-açúcar, na Zona da Mata Sul (Roda, 2004a; 2004b; 2004c; 2004d; 2005). De forma recente, poucos trabalhos foram realizados em relação às aves da Zona da Mata Norte de Pernambuco e Brejos de Altitude (Roda e Carlos, 2004; Pereira et al., 2005a; Farias e Castilho, 2006; Farias et al., 2007; Farias, 2009a).

A riqueza de espécies de aves para a caatinga de Pernambuco é de aproximadamente 270 espécies. O início dos inventários mais sistematizados nesse bioma se deu em 1976, na Reserva Biológica de Serra Negra, entre os municípios de Floresta, Ibimirim e Tacaratu (Coelho, 1987b). A caatinga pernambucana só teve outro inventário realizado 18 anos depois, por meio do Projeto de Conservação e Utilização Sustentável da Diversidade Biológica Brasileira (PROBIO) da Caatinga, com levantamento de aves em RPPNs localizadas nos municípios de Betânia e Floresta (Farias et al., 2005). Outros inventários foram realizados para o extremo oeste, em Lagoa Grande, Ouricuri, Parnamirim e Petrolina (Olmos et al., 2005), em diferentes fisionomias de caatinga (Farias, 2007a) e, recentemente, no Parque Nacional do Catimbau (Farias, 2009b). Nos últimos anos, trabalhos sobre novos registros de espécies e ampliações de distribuição geográfica têm contribuído para um conhecimento mais preciso das aves nesse bioma (Pacheco e Parrini, 2002; Dantas et al., 2007; Pereira et al., 2008).

\section{Abordagens recentes na ornitologia em Pernambuco}

Colecionar nomes locais das espécies de aves de uma determinada região pode ser considerado um início promissor para uma pesquisa etnoornitológica (Farias e Alves, 2007c). Assim, em Pernambuco, a sua primeira contribuição efetiva nessa linha de investigação se deu com a publicação de Aves de Pernambuco e seus nomes populares (Farias et al., 2000a). Destacam-se também as pesquisas realizadas por Brito e Pereira (2006) sobre os muitos nomes vernáculos locais para cinco espécies de aves, evidenciando 41 nomes locais para Tangara cayana, e Guimarães et al. (2006) que avaliaram o grau de conhecimento que as crianças de uma escola rural do município de Gravatá tinham sobre as aves daquela região. Outras investigações sobre nomenclatura e classificação etnoornitológica foram realizadas em Igarassu, na Zona da Mata Norte (Farias e Alves, 2007a), e suas implicações para o desenvolvimento de atividades pedagógicas no ensino formal local (Farias e Alves, 2007b).

Aavifauna em áreas urbanas também foi investigada (Farias et al., 2000b), destacando-se a análise da arborização pública como atrativo para as aves (Pereira et al., 2005b) e os registros de espécies de hábitos florestais e migratórios em praças e parques públicos das cidades de Recife e Olinda (Dantas et al., 2007), como Zimmerius gracilipes (Pereira et al., 2007) e Falco pereginus (Pereira et al., 2006a), ou ainda de novas e excepcionais ocorrências documentadas como Ciconia maguari, observada nos gramados do campus da UFPE (Pereira et al., 2006c).

Outro tema recentemente investigado foi o turismo de observação de aves. Em parceria com governos municipais, a OAP conseguiu realizar inventários de aves em Unidades de Conservação e engenhos na Zona da Mata e no Sertão (OAP, 2003a; 2003b), nos municípios de Moreno e Brejo da Madre de Deus. Nesse Estado, os limites e possibilidades para esse tipo de turismo foi estudado para Itamaracá, litoral norte de Pernambuco, indicando diretrizes para se instalar o turismo de observação de aves, assim como listas ornitológicas nos diversos ambientes daquela ilha, incluindo seis UCs (Farias e Castilho, 2006). Com o nítido crescimento desta atividade no mundo, muito se fala em estabelecer essa atividade ecoturística no Brasil, utilizando principalmente os argumentos sobre riqueza de espécies e ambientes. Em Pernambuco, pode-se notar que o estabelecimento desta atividade encontra fortes limites no que se refere ao espaço geográfico: ou são áreas particulares não protegidas e sem plano de manejo ou são UCs (Farias, 2007b). 
Atualmente, existem 76 UCs em Pernambuco (Uchôa-Neto e Silva, 2003). Dessas, apenas 33 tiveram sua avifauna inventariada (Tabela 1). Talvez muitas já não tenham condições de abrigar espécies endêmicas ou ameaçadas de extinção, dado o seu avançado estado de degradação. Em Pernambuco, estão registradas 45 espécies ameaçadas de extinção (Farias et al., 2008), de acordo com a lista do MMA (2003), e 51 são endêmicas da Mata Atlântica (Roda, 2002).

TABELA 1: Lista das Unidades de Conservação em Pernambuco que possuem inventários de aves.

\begin{tabular}{|c|c|c|}
\hline Unidade de Conservação & Município & Referências \\
\hline Estação Ecológica de Caetés & Paulista & Farias, 2009a \\
\hline Reserva Ecológica Mata do Engenho Uchoa & Recife & Farias et al., 2000b \\
\hline Parque Estadual de Dois Irmãos & Recife & Farias et al., 2000b \\
\hline Reserva Ecológica Mata de Tapacurá & São Lourenço da Mata & Coelho, 1987a \\
\hline Reserva Ecológica Mata do Toró & São Lourenço da Mata & Coelho, 1987a \\
\hline Reserva Ecológica Mata do Jardim Botânico & Recife & Farias et al., 2000b \\
\hline Reserva Ecológica Mata do Sistema Gurjaú & $\begin{array}{l}\text { Cabo de Santo Agostinho/ Moreno/ } \\
\text { Jaboatão dos Guararapes }\end{array}$ & Lyra-Neves et al., 2004c \\
\hline Reserva Ecológica Mata do Curado & Recife & Farias et al., 2000b \\
\hline Reserva Ecológica Mata de Dois Unidos & Recife & Farias et al., 2000b \\
\hline Reserva Ecológica Mata do Engenho Amparo & Itamaracá & Farias e Castilho, 2006 \\
\hline Reserva Ecológica Mata do Engenho Moreninho & Moreno & OAP, 2003a \\
\hline Reserva Ecológica Mata do Camucim & São Lourenço da Mata & Coelho, 1987a \\
\hline Reserva Ecológica Mata de Jaguaribe & Itamaracá & Farias e Castilho, 2006 \\
\hline Reserva Ecológica Mata do Engenho Macaxeira & Itamaracá & Farias e Castilho, 2006 \\
\hline Reserva Ecológica Mata Lanço dos Cações & Itamaracá & Farias e Castilho, 2006 \\
\hline Reserva Ecológica Mata do Engenho São João & Itamaracá & Farias e Castilho, 2006 \\
\hline Reserva Ecológica Mata de Santa Cruz & Itamaracá & Farias e Castilho, 2006 \\
\hline Reserva Ecológica Mata da Usina São José & Igarassu & Farias et al., 2007 \\
\hline Reserva Ecológica Mata do Outeiro do Pedro & São Lourenço da Mata & Coelho, 1987a \\
\hline Reserva Ecológica Mata do Engenho Tapacurá & São Lourenço da Mata & Coelho, 1987a \\
\hline Reserva Ecológica de São João da Várzea & Recife & Farias et al., 2000b \\
\hline Estação Ecológica de Tapacurá & São Lourenço da Mata & Coelho, 1987a \\
\hline Reserva Biológica de Serra Negra & Floresta/ Inajá/ Tacaratu & Coelho, 1987b \\
\hline Reserva Biológica de Saltinho & Tamandaré/ Rio Formoso & Roda, 2005 \\
\hline Parque Nacional do Catimbau & Buíque & Farias, 2009b \\
\hline Parque Natural Municipal João Vasconcelos Sobrinho & Caruaru e Altinho & Roda e Carlos, 2004 \\
\hline RPPN Fazenda Bituri & Brejo da Madre de Deus & OAP, 2003b \\
\hline RPPN Mauricio Dantas & Betânia/ Floresta & Farias et al., 2005 \\
\hline RPPN Santa Beatriz do Carnijó & Jaboatão dos Guararapes/ Moreno & OAP, 2003a \\
\hline RPPN Frei Caneca & Jaqueira & Roda, 2004a \\
\hline RPPN Cantidiano Valgueiro Carvalho Barros & Floresta & Farias et al., 2005 \\
\hline APA da Chapada do Araripe & Exu & Nascimento et al., 2000 \\
\hline APA de Guadalupe & $\begin{array}{l}\text { Sirinhaém/ Rio Formoso/ Tamanda- } \\
\text { ré/ Barreiros }\end{array}$ & Rodrigues et al., 2007 \\
\hline
\end{tabular}


Embora a produção do conhecimento sobre ornitologia tenha aumentado bastante nas últimas décadas no Brasil, é importante discutir se os recursos humanos especializados em ornitologia são suficientes para estudar uma avifauna tão rica e diversificada (Borges, 2008). Em Pernambuco, existe uma necessidade urgente de formação de biólogos ornitólogos que estejam habilitados em reconhecer as espécies em campo, principalmente através das vocalizações, o que facilitaria trabalhos de inventários e, principalmente, de ecologia de aves. Nesse Estado, os cursos de pós-graduação só realizaram 15 pesquisas com aves entre os anos de 1970 e 2005 (Borges, 2008) e, os seus principais ornitólogos, publicaram nos últimos dez anos aproximadamente 80 trabalhos, entre artigos, capítulos de livro e relatórios técnicos que contribuíram para o enriquecimento da ornitologia local. Dessa forma, pode-se afirmar que, embora a ornitologia em Pernambuco pareça bem desenvolvida, com professores da UFPE, UFRPE e ONGs desenvolvendo projetos nessa área e contando com um grupo de observadores de aves, realizando constantes inventários e estimulando o gosto por esta atividade, parece que ainda há muitos lugares e temas a serem investigados. É só observar a quantidade de novas informações que tem sido publicada anualmente sobre novas ocorrências de espécies ou documentações de aves da lista secundária local.

Dada à escassez de estudos sobre aves nas áreas de caatinga em Pernambuco, não resta à menor dúvida que o investimento em pesquisas neste hábitat seria o mais importante e prioritário nesse momento. Assim, inicialmente, deve-se continuar investindo em inventários, principalmente nas UCs, em propriedades particulares bem conservadas, nas serras interioranas e em áreas de caatinga. Por outro lado, nesse estado, muitas informações já foram coletadas, analisadas e publicadas, dando suporte suficiente para a indicação de Áreas Prioritárias para Conservação das aves (MMA, 2002) e Áreas Importantes para a Conservação de Aves (Bencke et al., 2006), de forma que ações efetivas para a conservação de espécies endêmicas ou ameaçadas de extinção também devem ser incentivadas.

\section{Referências}

Amaral, F. S. R; Silveira, L. F. 2004. Tinamus solitarius pernambucensis Berla, 1946 é sinônimo de Tinamus solitarius (Vieillot, 1819). Ararajuba, 12 (1): 33-41.

Antas, P. T. Z. 1983. Migration of Neartic shorebirds (Charadriidae and Scolopacidae) in Brazil: flyways and their different seazonal use. Wader Study Group Bulletin, 39: 52-56.

Azevedo-Júnior, S. M. 1992. Anilhamento de aves migratórias na Coroa do Avião, Igarassu, Pernambuco, Brasil. Caderno Ômega da Universidade Federal Rural de Pernambuco, Série Ciências Aquáticas, 3: 31-47.

Azevedo-Júnior, S. M. 1998. As aves do Canal de Santa Cruz, Pernambuco Brasil. Caderno Ômega da Universidade Federal Rural de Pernambuco, Série Biologia, 5: 35-50.

Azevedo-Júnior, S. M.; Dias, M. M.; Larrazábal, M. E. 2001a. Plumagens e mudas de Charadriiformes (Aves) no litoral de Pernambuco, Brasil. Revista Brasileira de Zoologia, 18 (3): 657 672.

Azevedo-Júnior, S. M.; Dias-Filho, M. M.; Larrazábal, M. E.; Fernandes, C. J. G. 2001c. Capacidade de vôo de quatro espécies de Charadriformes (Aves) capturadas em Pernambuco, Brasil. Revista Brasileira de Zoologia, 19 (1): 183-189.

Azevedo-Júnior, S. M.; Dias, M. M.; Larrazábal, M. E.; TelinoJúnior. W. R.; Lyra-Neves, R. M.; Fernandes, C. J. G. 2001b. Recapturas e recuperações de aves migratórias no litoral de Pernambuco, Brasil. Ararajuba, 9 (1): 33-42.

Azevedo-Júnior, S. M.; Larrazábal, M. E. 2002. Migração de Aves em Pernambuco. In: Tabarelli, M. \& Silva, J. M. C. (Orgs.). Diagnóstico da biodiversidade de Pernambuco. SECTMA/ Massangana, Recife, Brasil, p.623-630.

Azevedo-Júnior, S. M.; Larrazabal, M. E.; O. Pena. 2003. First record of Charadrius melodus Ord (Aves Charadriiformes) in Brazil. Revista Brasileira de Zoologia, 20 (3): 559-560.

Barnett, J. M.; Carlos, C. J.; Roda, S. A. 2005. Renewed hope for the threatened avian endemics of northeastern Brazil. Biodiversity and Conservation, 14: 2265-2274.

Bencke, G. A.; Maurício, G. N.; Develey, P. F.; Goerck, J. M. 2006. Áreas importantes para a conservação das aves no Brasil. Parte I - Estados do domínio da Mata Atlântica. SAVE Brasil, São Paulo, Brasil, 494pp.

Berla, H. F. 1946. Lista das aves colecionadas em Pernambuco, com descrição de uma subespécie N., de um alótipo fêmea e notas de campo. Boletim do Museu Nacional, 65: 1-35.

Borges, S. H. 2008. A importância do ensino de pós-graduação na formação de recursos humanos para o estudo da biodiversidade no Brasil: um estudo de caso na ornitologia. Biota Neotropica, 8 (1): 21-27.

Brito, M. T.; Pereira, G. A. 2006. Relação das cinco espécies de aves do estado de Pernambuco com mais nomes populares. Atualidades Ornitológicas, 129: 14-15.

Carlos, C. J.; Fedrizzi, C. E.; Azevedo-Júnior, S. M. 2005. Notes on some seabirds of Pernambuco state, north-east Brazil. Bulletin of British Ornithologists Club, 125 (2): 140-147.

Coelho, A. G. M. 1979. As aves da Estação Ecológica de Tapacurá, Pernambuco. Notulae Biologicae, 2: 1-18. 
Coelho, A. G. M. 1987a. Novas informações sobre a avifauna da Estação Ecológica de Tapacurá - Pernambuco. Recife: Publicação avulsa, 1: 1-16.

Coelho, A. G. M. 1987b. Aves da Reserva Biológica de Serra Negra (Floresta-PE), lista preliminar. Recife: Publicação avulsa, 2: 1-8.

Dantas, S. M.; Pereira, G. A.; Farias, G. B.; Brito, M. T. B.; Periquito, M. C.; Pacheco, G. L.; Vasconcelos, E. S. T. 2007. Registros relevantes de aves para o estado de Pernambuco, Brasil. Revista Brasileira de Ornitologia, 15 (1): 113-115.

Farias, G. B. 2007a. Avifauna em quatro áreas de caatinga strictu senso no centro-oeste de Pernambuco, Brasil. Revista Brasileira de Ornitologia, 15 (1) 103-110.

Farias, G. B. 2007b. A observação de aves como possibilidade ecoturística. Revista Brasileira de Ornitologia, 15: 474-477.

Farias, G. B. 2009a. Variação temporal em uma comunidade de aves em área de Mata Atlântica na Estação Ecológica de Caetés, Pernambuco, Brasil. Atualidades Ornitológicas, 147: 40-45.

Farias, G. B. 2009b. Aves do Parque Nacional do Catimbau, Buíque, Pernambuco, Brasil. Atualidades Ornitológicas, 147: 4-7.

Farias, G. B.; Alves, A. G. C. 2007a. Nomenclatura e classificação etnoornitológica em fragmentos de Mata Atlântica em Igarassu, Região Metropolitana do Recife, Pernambuco. Revista Brasileira de Ornitologia, 15 (3): 358-366.

Farias, G. B.; Alves, A, G. C. 2007b. Conhecimento prévio sobre a avifauna por alunos do Ensino Fundamental numa escola pública na Região Metropolitana do Recife: Em busca de uma prática pedagógica culturalmente apropriada. In: Albuquerque, U. P; Alves, A. G. C. \& Araújo, T. A. S. (Orgs). Povos e paisagens: Etnobiologia, etnoecologia e biodiversidade no Brasil. NUPEEA/ UFRPE, Recife, Brasil, p.48-59.

Farias, G. B.; Alves, A, G. C. 2007c. É importante pesquisar o nome local das aves? Revista Brasileira de Ornitologia, 15 (3): 403408.

Farias, G. B.; Alves, A, G. C.; Lins e Silva, A. C. B. 2007. Riqueza de aves em cinco fragmentos de Floresta Atlântica na Zona da Mata Norte de Pernambuco, Brasil. Biotemas, 20 (4): 111-122.

Farias, G. B.; Brito, M. T.; Pacheco, G. L. 1995. Lista Preliminar das aves do Estado de Pernambuco. Observadores de Aves de Pernambuco, Recife, Brasil, 44pp.

Farias, G. B.; Brito, M. T.; Pacheco, G. L. 2000a. Aves de Pernambuco e seus nomes populares. Editora Universitária da UFPE, Recife, Brasil, 56pp.

Farias, G. B.; Brito, M. T.; Pacheco, G. L. 2000b. Aves. In: Vasconcelos, R. F. A. \& Bezerra, O. G. (Eds). Atlas ambiental da cidade do Recife. PCR/ SPUMA/ LICEU, Recife, Brasil, p.73-80.

Farias, G. B.; Brito, M. T.; Pacheco, G. L. 2002. Registros ornitológicos de Pernambuco. Observadores de Aves de Pernambuco, Recife, Brasil, 67pp.

Farias, G. B.; Brito, M. T.; Pacheco, G. L.; Telino-Júnior, W. R.; Lyra-Neves, R. M. 1994. Nota sobre a ocorrência da "Pombaantártica" Chionis alba (Charadriiformes-Chionididae) para o estado de Pernambuco. Resumos do IV Congresso Brasileiro de Ornitologia, Recife, Brasil, p.70.

Farias, G. B.; Castilho, C. J. M. 2006. Observação de aves e ecoturismo em Itamaracá(PE): Instrumentos para o desenvolvimento sustentável. Sociedade \& Natureza, 18 (35): 35-53.
Farias, G. B.; Pereira, G. A.; Silva, W. A. G. 2008. Lista das aves de Pernambuco. Observadores de Aves de Pernambuco, Recife, Brasil, 40pp.

Farias, G. B.; Silva, W. A. G.; Albano, C. G. 2005. Diversidade de aves em áreas prioritárias para conservação da Caatinga. In: Araújo, F. S.; Rodal, M. J. \& Barbosa, M. R. V. (Orgs). Análise das variações da biodiversidade do bioma caatinga: Suporte e estratégias regionais de conservação. MMA, Brasília, Brasil, p.203-226.

Fedrizzi, C. E.; Azevedo-Júnior, S. M.; Larazzábal, M. E. L. 2004. Body mass and acquisition of breeding plumage of wintering Calidris pusilla (Linnaeus) (Aves, Scolopacidae) in the coast of Pernambuco, north-eastern Brazil. Revista Brasileira de Zoologia, 21 (2): 249-252.

Forbes, W. A. 1881. Eleven weeks in North-eastern Brazil. Ibis, 5: 312-362.

Guimarães, E.; Pereira, G. A.; Brito, M. T.; Silva, R. M.; Dantas, S. M. 2006. Percepção da avifauna por alunos de uma escola rural no Agreste do estado de Pernambuco, Brasil. Atualidades Ornitológicas, 132: 12-13.

Ihering, H.; Ihering, R. 1907. As aves do Brazil. Catálogos da Fauna Brazileira, vol. 1. Museu Paulista, São Paulo, Brasil, 485pp.

Kadletz, T. 1933. Synopse das aves do estado de Pernambuco. Revista do Gymnasio Pernambucano, 2: 29-54.

Lamm, D. W. 1948. Notes on the birds of the states of Pernambuco and Paraíba, Brazil. Auk, 65: 261-283.

Lyra-Neves, R. M.; Azevedo-Júnior, S. M.; Telino-Júnior, W. T.; 2004b. Monitoramento do maçarico-branco, Calidris alba (Pallas) (Aves, Scolopacidae), através de recuperações de anilhas coloridas, na Coroa do Avião, Igarassu, Pernambuco, Brasil. Revista Brasileira de Zoologia, 21 (2): 319-324.

Lyra-Neves, R. M.; Dias, M. M.; Azevedo-Júnior, S. M.; TelinoJúnior, W. T.; Larrazábal, M. E. 2004c. Comunidade de aves da Reserva Estadual de Gurjaú, Pernambuco, Brasil. Revista Brasileira de Zoologia, 21 (3): 581-592.

Lyra-Neves, R. M., Farias, A. M. I.; Telino-Júnior, W. R. 2003. Ecological relationships between feather mites (Acari) and wild birds of Emberizidae (Aves) in a fragment of Atlantic Forest in northeastern Brazil. Revista Brasileira de Zoologia, 20 (3): 481485.

Lyra-Neves, R. M.; Farias, A, M. I.; Telino-Júnior, W. R. 2005. Interações entre Phthiraptera (Insecta) e aves (Emberizidae) de Mata Atlântica, Pernambuco, Brasil. Ornithologia, 1 (1): 43-47.

Lyra-Neves, R. M.; Farias, A. M. I.; Telino-Júnior, W. R.; Botelho, M. C. N.; Lima, M. C. A. 2000. Ectoparasitismo em aves silvestres (Passeriformes- Emberizidae) de Mata Atlântica, Igarassu, Pernambuco. Melopsittacus, 3 (2): 64-71.

Lyra-Neves, R. M.; Oliveira, M. A. B. ; Telino-Júnior, W. T.; Santos, E. M. 2007. Comportamentos Interespecíficos entre Callithrix jacchus (Linnaeus) (Primates, Callitrichidae) e algumas aves de Mata Atlântica, Pernambuco, Brasil. Revista Brasileira de Zoologia, 24 (3): 709-716.

Lyra-Neves, R. M.; Telino-Júnior, W. T.; Dias, M. M.; AzevedoJúnior, S. M. 2004a. Primeiro registro de Myiarchus tuberculifer (Lafresnaye \& d'Orbiny) (Aves, Tyrannidae) para o Estado de Pernambuco, Brasil. Revista Brasileira de Zoologia, 21 (2): 405 407. 
Magalhães, V. S.; Azevedo-Júnior, S. M.; Lyra-Neves, R. M.; Telino-Júnior, W. R.; Souza, D. P. 2007. Biologia de aves capturadas em um fragmento de Mata Atlântica, Igarassu, Pernambuco, Brasil. Revista Brasileira de Zoologia, 24 (4): 950-964.

Marcgrave, J. 1942. História Natural do Brasil. Museu Paulista, São Paulo, Brasil, 293+CIVpp.

Melo, M. 1960. Aves de Pernambuco, nomes vulgares e científicos. Revista do Instituto Arqueológico Histórico e Geográfico Pernambucano, 45: 265-300.

MMA - Ministério do Meio Ambiente. 2002. Avaliação e identificação de áreas e ações prioritárias para a conservação, utilização sustentável e repartição dos benefícios da biodiversidade nos biomas brasileiros. MMA/ SBF, Brasília, Brasil, 404pp.

MMA - Ministério do Meio Ambiente. 2003. Instrução Normativa $\mathrm{n}^{\circ} 3$ de 27 de maio de 2003. Lista das espécies da fauna brasileira ameaçadas de extinção. Diário Oficial da República Federativa do Brasil, Brasília, Brasil.

Nascimento, J. L. X.; Nascimento, I. L. S.; Azevedo-Júnior, S.M. 2000. Aves da Chapada do Araripe (Brasil): biologia e conservação. Ararajuba, 8 (2): 115-125.

OAP-Observadores de Aves de Pernambuco. 2003a. Levantamento preliminar da avifauna no município do Moreno - Pernambuco. Disponível em $<$ http://www.oap.org.br/publica.htm>. Acesso em 10 de agosto de 2008.

OAP - Observadores de Aves de Pernambuco. 2003b. Levantamento preliminar da avifauna no município do Brejo da Madre de Deus, Pernambuco. Disponível em $<$ http://www. oap.org.br/publica.htm>. Acesso em 10 de agosto de 2008.

Olmos, F.; Silva, W. A. G.; Albano, C. G. 2005. Aves em oito áreas de caatinga no sul do Ceará e oeste de Pernambuco, Nordeste do Brasil: composição, riqueza e similaridade. Papéis Avulsos de Zoologia, 45: 179-199.

Pacheco, J. F. 2000. A ornitologia descobre o sertão: Um balanço do conhecimento da avifauna na Caatinga dos primórdios aos anos 1950. In: Straube, F. C.; Argel-de-Oliveira. M. M. \& CândidoJúnior, J. F. (Eds). Ornitologia brasileira no Século XX. Editora Popular, Curitiba, Brasil, p.11-70.

Pacheco, J. F.; Parrini, R. 2002. Alguns registros relevantes de aves para o estado de Pernambuco. Atualidades Ornitológicas, 109: 7.

Pereira, G. A.; Coelho, G.; Dantas, S. M.; Roda, S. A.; Farias, G. B.; Roda, S. A.; Brito, M. T. B.; Pacheco, G. L. 2006a. Ocorrências e hábitos alimentares do falcão-peregrino Falco peregrinus no estado de Pernambuco, Brasil. Revista Brasileira de Ornitologia, 14 (4): 435-439.

Pereira, G. A.; Dantas, S. M.; Periquito, M. C. 2006b. Possível registro de Leptodon forbesi no estado de Pernambuco, Brasil. Revista Brasileira de Ornitologia, 14 (4): 441-444.

Pereira, G. A.; Farias, G. B.; Girão, W.; Periquito, M. C. 2006c. Primeiro registro documentado de Ciconia maguari (Aves: Ciconiidae) para o Estado de Pernambuco. Revista Brasileira de Ornitologia, 14 (2): 153-154.

Pereira, G. A.; Dantas, S. M.; Roda, S. A. 2005a. Aves da Mata de Aldeia, Camaragibe, Pernambuco. Relatório Técnico. CEPAN, Recife, Brasil, 17pp. Disponível em < http://www.cepan.org.br/>. Acesso em 30 de setembro de 2008.
Pereira, G. A.; Monteiro, C. S.; Campelo, M. A.; Medeiros, C. 2005b. $\mathrm{O}$ uso de espécies vegetais, como instrumento de biodiversidade da avifauna silvestre, na arborização pública: o caso do Recife. Atualidades Ornitológicas, 125: 10.

Pereira, G. A.; Periquito, M. C.; Dantas, S. M. 2007. Presença do poiaeiro-de-pata-fina, Zimmerius gracilipes (Aves: Tyrannidae), em áreas urbanas na Região Metropolitana do Recife, Pernambuco, Brasil. Revista Brasileira de Ornitologia, 15 (3): 443-444.

Pereira, G. A.; Whittaker, A.; Whitney, B.; Zimmer, K. J.; Dantas, S. M.; Roda, S. A.; Bevier, L. R.; Coelho, G.; Hoyer, R. C.; Albano, C. 2008. Novos registros relevantes de aves para o estado de Pernambuco, Brasil, incluindo novos registros para o Estado. Revista Brasileira de Ornitologia, 16 (1): 47-53.

Pinto, O. M. O. 1938. Catálogo das aves do Brasil e lista dos exemplares que as representam no Museu Paulista. $1^{\circ}$ parte. Aves não Passeriformes e Passeriformes não Oscines excluída a Fam. Tyrannidae e seguintes. Revista do Museu Paulista, 22: 1-566.

Pinto, O. M. O. 1940. Aves de Pernambuco. Breve ensaio retrospectivo com lista de exemplares coligidos e descrição de algumas formas novas. São Paulo: Arquivo de Zoologia do Estado de São Paulo, 1 (5): 219-282.

Pinto, O. M. O. 1944. Catálogo das aves do Brasil, $2^{\text {a }}$ parte. Ordem Passeriformes (continuação): superfamília Tyrannoidea e Subordem Passeres. Secretaria de Agricultura de São Paulo, São Paulo, Brasil, 326pp.

Reiser, O. 1925. Vögel. In: Ergebnisse der Zoolog: Expedition der Akademie der Wissenschaften nach Nordostbrasilien im Jahre 1903. Kaiserlichen Akademie der Wissenschaften, Wien, Aústria, p.107-252.

Roda, S. A. 2002. Aves endêmicas e ameaçadas de extinção no estado de Pernambuco, In: Tabarelli, M. \& Silva, J. M. C. (Orgs.). Diagnóstico da Biodiversidade de Pernambuco. SECTMA/ Massangana, Recife, Brasil, p.537-555.

Roda, S. A. 2004a. Composição e conservação de aves em ambientes fragmentados na floresta Atlântica nordestina. Relatório Técnico. CEPAN, Recife, Brasil, 68pp. Disponível em < http://www.cepan.org.br/>. Acesso em 30 de setembro de 2008.

Roda, S. A. 2004b. Lista preliminar das aves da usina Trapiche, Serinhanhém, PE. Relatório Técnico. CEPAN, Recife, Brasil, 35pp. Disponível em $<$ http://www.cepan.org.br/>. Acesso em 30 de setembro de 2008.

Roda, S. A. 2004c. Aves da Fazenda Morim, São José da Coroa Grande, PE. Relatório Técnico. CEPAN, Recife, Brasil, 32pp. Disponível em <http://www.cepan.org.br/>. Acesso em 30 de setembro de 2008.

Roda, S. A. 2004d. Aves da Usina Cucaú, PE. Relatório Técnico. CEPAN, Recife, Brasil, 30pp. Disponível em $<$ http://www.cepan. org.br/>. Acesso em 30 de setembro de 2008.

Roda, S. A. 2005. Aves da Reserva Biológica de Saltinho, PE. Relatório Técnico. CEPAN, Recife, Brasil, 42pp. Disponível em $<$ http://www.cepan.org.br/>. Acesso em 30 de setembro de 2008.

Roda, S. A.; Carlos, C. J. 2003. New records for some poorly know birds of Atlantic forest of northeastern Brazil. Cotinga, 20: 17-20.

Roda, S. A.; Carlos, C. J. 2004. Composição e sensitividade da avifauna dos brejos de altitude do estado de Pernambuco. In: Pôrto, K.; Cabral, J. \& Tabarelli, M. (Orgs.). Brejos de altitude: História 
natural, ecologia e conservação. MMA, Brasília, Brasil, p.203219.

Roda, S. A.; Carlos, C. J.; Rodrigues, R. C. 2003. New and noteworthy records for some endemic and threatened birds in the Atlantic forest of northeastern Brazil. Bulletin of the Brithish Ornithological Club, 123 (4): 227-236.

Roda, S. A.; Farias, A. M. I. 1999. Ácarlos plumícolas em aves Passeriformes da Zona da Mata Norte de Pernambuco, Brasil. Revista Brasileira de Zoologia, 16 (3):879-886.

Roda, S. A.; Pereira, G. A. 2006. Distribuição recente e conservação de aves de rapinas florestais do Centro Pernambuco. Revista Brasileira de Ornitologia, 14 (4): 331-344.

Rodrigues, R. C.; Araújo, H. F. P.; Lyra-Neves, R. M.; Telino-Júnior, W. R.; Botelho, M. C. N. 2007. Caracterização da Avifauna na área de Proteção Ambiental de Guadalupe, Pernambuco. Ornithologia, 2: 47-61.

Rosado, V.; Silva, A. C. 1973. Louis Jacques Brunet: naturalista viajante. CERN, Natal, Brasil, 341pp.

Sick, H. 1997. Ornitologia brasileira. Editora Nova Fronteira, Rio de Janeiro, Brasil, 862pp.

Silva, J. M. C.; Coelho, G.; Gonzaga, L. P. 2002. Discovered on the brink of extinction: A new species of Pygmy-Owl (Strigidae: Glaucidium) from Atlantic Forest of northeastern Brazil. Ararajuba, 10 (3): 123-130.

Silveira, L. F.; Olmos, F.; Roda, S. A.; Long, A. J. 2003. Notes on the Seven-coloured Tanager Tangara fastuosa (Lesson, 1831) in North-east Brazil. Cotinga, 20: 82-88.

Stotz, D. F.; Fitzpatrick, J. W.; Parker, T.A. III.; Moskovits, D. K. 1996. Neotropical birds: Ecology and conservation. University of Chicago Press, Chicago, USA, 478pp.

Speich, S. M. 1999. In Memoriam: Donald Wakeham Lamm, 19141996. Auk, 116 (3): 818.

Teixeira, D. M. 1992. As fontes do Paraíso. Um ensaio sobre a ornitologia no Brasil holandês (1624-1654). Revista Nordestina de Biologia, 7 (1/2): 1-149.

Teixeira, D. M.; Nacinovic, J. B.; Luigi, G. 1988. Notes on some birds of northeastern Brazil (3). Bulletin of British Ornithologists Club, 108 (2): 75-79.
Teixeira, D. M.; Nacinovic, J. B.; Luigi, G. 1989. Notes on some birds of northeastern Brazil (4). Bulletin of British Ornithologists Club, 109 (3): 152-157.

Teixeira, D. M.; Nacinovic, J. B.; Pontual, F. B. 1987. Notes on some birds of northeastern Brazil (2). Bulletin of British Ornithologists Club, 107 (4): 151-157.

Teixeira, D. M.; Nacinovic, J. B.; Tavares, M. S. 1986. Notes on some birds of northeastern Brazil. Bulletin of British Ornithologists Club, 106 (2): 70-74.

Teixeira, D. M.; Otoch, R.; Luigi, G.; Raposo, M. A.; Almeida, A. C. C. 1993. Notes on some birds of northeastern Brazil (5). Bulletin of the British Ornithologists Club, 113 (1): 48-52.

Telino-Júnior, W. R.; Azevedo-Júnior, S. M.; Lyra-Neves, R. M. 2003. Censo de aves migratórias (Charadriidae, Scolopacidae e Laridae) na Coroa do Avião, Igarassu, Pernambuco, Brasil. Revista Brasileira de Zoologia, 20 (3): 451-456.

Telino-Júnior, W. R.; Dias, M. M.; Azevedo-Júnior, S. M.; LyraNeves, R. M.; Larrazábal, M. E. L. 2005. Estrutura trófica da avifauna na Reserva Estadual de Gurjaú, Zona da Mata Sul, Pernambuco, Brasil. Revista Brasileira de Zoologia, 22 (4): 962973.

Telino-Júnior, W. R.; Lyra-Neves, R. M.; Carneiro, R. S. 2000. Observações de Touit surda (Psittacidae) em fragmentos florestais de Pernambuco, Brasil. Melopsitacus, 3 (4): 159-165.

Uchôa-Neto, C. A. M.; Silva, J. M. C. 2002. Análise de representatividade das Unidades de Conservação no estado de Pernambuco. In: Tabarelli, M. \& Silva, J. M. C. (Orgs). Diagnóstico da Biodiversidade de Pernambuco. SECTMA/ Massangana, Recife, Brasil, p.707-716.

Vooren, C. M.; Brusque L. F. 1999. Diagnóstico sobre aves do ambiente costeiro do Brasil. In: Avaliação e ações prioritárias para a conservação da biodiversidade da Zona Costeira e Marinha. Programa Nacional da Diversidade Biológica. Disponível em $<$ http://www.bdt.org.br/workshop/costa/aves/>. Acesso em 14 de julho de 2008 . 